\title{
Report of 12 cases of ankylosing spondylitis patients treated with Tripterygium wilfordii
}

\author{
Wei Ji • Juan Li • Yue Lin • Ya-nan Song • \\ Miaojia Zhang • Yao Ke $\cdot$ Yile Ren $\cdot$ Xiaohu Deng • \\ Jianglin Zhang $\cdot$ Feng Huang $\cdot$ David Yu
}

Received: 18 May 2009/Revised: 27 February 2010 / Accepted: 20 May 2010 / Published online: 19 June 2010

(C) The Author(s) 2010. This article is published with open access at Springerlink.com

\begin{abstract}
Objective Description of the clinical response of 12 consecutive cases of disease-active ankylosing spondylitis (AS) treated with the herbal medicine Tripterygium wilfordii Hook f (TwHf; lei gong teng, thunder god vine), which has been reported in controlled studies to be effective in rheumatoid arthritis (RA).

Methods The clinical status of 12 patients with active AS who were started on 60 mgday $^{-1}$ of a commercial tablet preparation of TwHf extract. were monitored at weeks 1, 3, and 6 . Results Compared to baseline, there was significant improvement in mean values of physician assessment, Bath ankylosing spondylitis disease activity index (BASDAI), Bath ankylosing
\end{abstract}

\footnotetext{
W. Ji $(\bowtie)$

Department of Rheumatology, Jiangsu Province Hospital of Traditional Chinese Medicine,

155 HanZhong Road,

Nanjing, Jiangsu, China, 210029

e-mail: weiweiji1103@yahoo.com.cn

J. Li $\cdot$ Y. Lin $\cdot$ Y.-n. Song

Department of Rheumatology, Jiangsu Province Hospital of Traditional Chinese Medicine,

Nanjing, Jiangsu, China

M. Zhang $\cdot$ Y. Ke $\cdot$ Y. Ren

Department of Rheumatology, The First Affiliated Hospital of the Nanjing Medical University,

Nanjing, Jiangsu, China

X. Deng $\cdot$ J. Zhang $\cdot$ F. Huang

Department of Rheumatology, Chinese PLA General Hospital, Beijing, China

D. $\mathrm{Yu}$

Department of Medicine, University of California Los Angeles, Los Angeles, CA, USA
}

spondylitis functional index (BASFI), and Bath ankylosing spondylitis global score (BAS-G) at weeks 3 and 6, with no changes in liver enzymes or complete blood count (CBC). Conclusion A placebo-controlled double-blind study for Tripterygium is warranted. Until then, this particular report should be considered as case reports and not an endorsement of the use of Tripterygium in clinical practice.

Keywords Ankylosing spondylitis · Lei gong teng · Tripterygium wilfordii Hook $\mathrm{f}$

\section{Introduction}

One of the medicinal herbs widely used used in China for the treatment of autoimmune and inflammatory diseases is the vine Tripterygium wilfordii Hook $\mathrm{f}$ (TwHf), also known by the descriptive names lei gong teng, thunder god vine, and seven-step vine [1-3]. A rapid pubmed search for the keywords "Tripterygium" plus "inflammatory" yields a total of 120 publications, while a search for "Tripterygium" and "lupus" yields 30 publications. More than 21 autoimmune and inflammatory diseases have been reported to have been treated with extracts of Tripterygium. At least five pharmaceutical companies in China are marketing extracts of $T$. wilfordii in tablet forms and have been approved by the China State Food and Drug Administration for use in rheumatoid arthritis (RA), systemic lupus erythematosus (SLE), and ankylosing spondylitis (AS). They are being used by many rheumatologists and nephrologists who practice traditional medicine in China [1-3]. For example, for the Jiangsu Province Hospital of Traditional Chinese Medicine in Nanjing, the number of tablets of T. wilfordii prescribed in 2008 was 620,000 , the average per month in 2009 has been about 50,000. At least 
three controlled double-blind studies for RA with promising results have been reported, one carried out in China and the others in North America [4-7]. Beneficial effects were observed in RA as early as 4 weeks after initiation of Tripterygium. In vitro and animal model studies suggest that Tripterygium is anti-inflammatory and immunosuppressive, partly by suppressing the generation of proinflammatory cytokines (reviewed in Ref. [1]).

However, no pubmed abstract-available studies have been reported on AS. Search for literature in a Chinese language database showed that the earliest report of the use of T. wilfordii in AS was in 1981 [8]. Since then, at least four other Chinese language papers have been published, comprising a total of 175 AS patients (reviewed in Ref. [3]). The efficacy of $T$. wilfordii was broadly described as being nearly $90 \%$ effective. However, none of these Chinese language papers assessed patients with internationally standardized evaluation instruments. AS is an inflammatory arthritis affecting mainly the axial skeleton. Like RA, AS also respond to treatment by nonsteroidal antiinflammatory drugs (NSAID), sulfasalazine, and, more dramatically, to tumor necrosis factor (TNF) blockers. Unlike RA, there is no good evidence that it responds well to methotrexate, leflunomide, or hydroxychloroquine [9, 10]. This paper describes 12 consecutive disease-active AS patients who were started on Tripterygium and evaluated with internationally approved evaluation instruments. This paper is a case report and not a randomized placebocontrolled study. In our analysis, we attempted to evaluate the possible significance of response to Tripterygium by comparing the Tripterygium results to those of 26 AS patients on placebo in a controlled study for etanercept.

\section{Materials and methods}

Patients receiving Tripterygium were those attending the Jiangsu Province Hospital of Traditional Chinese Medicine, Jiangsu, Nanjing, China. The study and informed consent followed the guidance of the Hospital Institutional Research Board (approval ref. no. 2009NL-1501). The study was designed to arbitrarily include all consecutive patients within a period of 4 months who satisified the following criteria: They satisfied the 1984 Modified New York Criteria for AS [11]. Their pelvis X-ray showed a sacroiliitis score of $\geq 4$ when combining scores of both right and left sides. Their scores for Bath ankylosing spondylitis disease activity index (BASDAI) and for spinal pain were $\geq 40$ on a scale of 0 to 100 . They had failed NSAID. Of the 12 patients, eight had failed $\geq 1$ month NSAID, while $8 / 12$ had also failed $\geq 3$ months of at least one disease-modifying antirheumatic drugs (DMARD). All prospective patients were advised of the antifertility effect of Tripterygium. Following the standard practice in this institute, excluded from Tripterygium treatment, were those patients with potential family planning, or being positive for hepatitis antigens, or showed elevated liver enzymes in blood tests. A general evaluation was also carried out to exclude those with significant co-morbidities. None of the AS patients in this case report had psoriasis or active uveitis or had used corticosteroids within the past 1 month. Patients who were on NSAID and DMARD were allowed to continue their medications with no increase in dosages. The Tripterygium used in these 12 patients was manufactured by the Zhe Jiang De En De Pharmaceutical (Zhejiang, China), the supplier for this institute. The lot number of the tablets was 0802102. The dose of the Tripterygium was $20 \mathrm{mg} 3$ times daily. Evaluations were carried out at entry of study as well as at weeks 1,3 , and 6 . After finishing the 6 weeks of observation, patients were followed by their own personal physicians and not by the investigators.

The placebo patients were derived from a randomized double-blind controlled study of etanercept carried in the Chinese PLA General Hospital, Beijing, China. Placebo was administered subcutaneously twice a week. The selection of patients according to their disease severity was similar to those of the Tripterygium patients in that the BASDAI score at baseline should be $\geq 40$ (scale 0-100). Details were similar to those of another placebo/etanercept study carried out also in the Chinese PLA General Hospital reported recently [12]. This study also followed the guidelines of the local and national Institutional Review Boards.

Evaluations included physician and patient selfassessment (Bath ankylosing spondylitis global score, BAS-G) over the past week, joint count for swelling, BASDAI, Bath ankylosing spondylitis functional index (BASFI), and ranges of motion included in the Bath ankylosing spondylitis metrology index (BASMI) [13-16]. Endpoints of clinical response were expressed as ASAS20 and ASAS40 [17]. Blood tests included erythrocyte sedimentation rate (ESR), C-reactive protein (CRP), complete blood count (CBC), and liver tests. For assessment of possible side effects, in addition to laboratory tests and general review of systems, also was enquired patients' own assessment of possible side effects.

For statistical evaluations, the overall significance of each parameter of clinical response was first tested by the Friedman test. Those with $p$ values $<0.001$ were further evaluated by the Wilcoxon signed-rank test. Other statistical tests used were the $t$ test for unequal variances and the chi-square test. All $p$ values were corrected for multiple testing by multiplying the values with 20 (Bonferroni factor for these data sets). Values were considered significant if the corrected $p$ values were $\leq 0.05$. One evaluation datum was missing and was carried forward from the previous week. Because 8 of the 12 Tripterygium patients were on 
DMARD, no analysis was carried out to compare effect of Tripterygium on patients on DMARD to those not on DMARD.

\section{Results}

The demographics and clinical characteristics of the 12 patients were listed in Table 1. The mean age was $36.2 \pm$ 10.9 years, mean duration of disease $6.8 \pm 4.3$ years, and mean BASDAI value $52 \pm 11.4$ (0-100 scale). While all patients complained of spinal pain, swollen joints were detected in only three. HLA-B27 was tested in ten patients and was positive in nine. All patients were carefully followed up for 6 weeks, except patient \#6 who did not return for the 6th week apparently because of lack of response. Patient numbering was consecutive except \#5 which did not exist.

By week 3 of treatment, the percent of patients showing ASAS20 and ASAS40 response were $91.7 \%$ and 33.3\%, respectively. These appeared to be sustained to week 6 (Fig. 1). Statistically significant improvement as assessed first by Friedman test $(p<0.001)$, followed by Wilcoxon signed-rank test, followed by correction for multiple testing was observed with BAS-G, BASDAI, and BASFI starting at week 1 (Fig. 2). For BAS-G and BASDAI, the improvement at week 6 exceeded those at week 3 . Statistically significant improvement for physician assessment was observed at weeks 3 and 6 . Although there was improvement in BASMI, it was not statistically significant after correction for multiple testing. At week 6, the ESR decreased from $26.8 \pm 16.7$ at week 0 to $9.8 \pm 5.8$ ( $p=0.007$ before Bonferroni correction). The decrease in CRP became statistically significant at week $6(p=0.04$ after Bonferroni correction; Fig. 2).

There were no clinically significant changes in $\mathrm{CBC}$ and liver function tests. None of the patients reported of any side effects such as headache or abdominal discomfort.

In a separate study, a total of 26 patients were given placebo in a randomized double-blind controlled study for etanercept. In this placebo group, NSAID, DMARD, and corticosteroids were also maintained at the same doses as in the baseline. The mean age of these patients was $30.1 \pm 7.7$ years, and the mean duration of disease was $9.7 \pm 6.1$ years. Out of 26 patients, 25 were male. The mean BASDAI, BASFI, and BAS-G values are shown in Table 2. At the entry of study, there were no statistically significant differences between this placebo group and the Tripterygium group in age, duration of disease, BASDAI, and BASFI. In the placebo group, after 6 weeks, even on placebo, there were $10-20 \%$ decrease in the values of BASDAI, BASFI, and BAS-G. These decreases were statistically significant in BASDAI and
BAS-G $(p=0.0001$ and 0.01$)$, but not BASFI $(p=0.09$; Table 2). The decreases in values of BASDAI, BASFI, and BAS-G at week 6 of the Tripterygium group were about $60 \%$, and compared to the corresponding values of the placebo group, they were significantly lower in the Tripterygium group ( $p=0.0001$ for all three parameters; Table 2 and Fig. 1). At week 6, the percent of patients in the placebo group who achieved ASAS20 and ASAS40 were $30.8 \%$ and $3.8 \%$, respectively. These were significantly less than the corresponding values in the Tripterygium group $(p<0.01$ and $<0.001$, respectively).

\section{Discussion}

Extracts of T. wilfordii are being distributed in tablet form as patent medicines by at least six pharmaceutical companies in China. The cost is about US $\$ 0.50$ per day, which is much lower than that of the biologics. They are widely used by physicians practicing traditional medicine and accepted by patients in China as standard treatment for several inflammatory and autoimmune diseases, especially RA, AS, SLE, and nephritis. A large number of publications, mostly in the Chinese language, have been reported [2-4, 6]. It is also being used for AS. A total of 175 AS patients treated with $T$. wilfordii have been reported in Chinese language papers [3]. None of them used internationally standardized evaluation instruments. Nevertheless, the drug was reported as being $90 \%$ effective. Since a major side effect of this drug is antifertility in male and amenorrhea in female, and because many AS patients are in the reproductive age, it is imperative to understand the risk/benefit ratio more precisely before continuing such indiscriminate use [18]. Although the Jiangsu Province Hospital of Traditional Chinese Medicine has been using this drug routinely for AS resistant to conventional therapies, this study is also the first in this institute to use internationally standardized evaluation instruments to describe 12 consecutive disease-active AS patients. The particular commercial preparation used in this study was one which has been used in this hospital for the past 5 years. The compilations of results of these 12 patients do show significant improvement in BAS-G, BASDAI, BASFI, and physician assessment. One needs to be cautioned that all these are subjective parameters. Since these are case reports, no placebo-controlled data were collected. In a recent placebo-controlled study of etanercept in AS, also carried out in China, data are available from 26 patients who received 6 weeks treatment with placebo. At week 6 , only $31 \%$ of those placebo-treated patients showed an ASAS20 response. The results from three separate published placebo-controlled study for TNF $\alpha$ carried out in Caucasian countries also showed that the ASAS20 response for placebo at weeks $4-8$ are about $20 \%$ 
Table 1 Demographics and patient characteristics at entry
There is no patient \#5. Units of measurements are in text.

MTX was at $10 \mathrm{mg}$ week $^{-1}$, sulfasalazine at $1.5 \mathrm{gday}^{-1}$, and thalidomide at $100 \mathrm{mg} \mathrm{day}^{-1}$.

\begin{tabular}{|c|c|c|c|c|c|}
\hline Designation & Age & Gender & Years of AS & \multicolumn{2}{|l|}{ DMARD } \\
\hline Patient 1 & 31 & $\mathrm{~F}$ & 2 & \multicolumn{2}{|l|}{ Methotrexate (MTX) } \\
\hline Patient 2 & 42 & M & 13 & \multicolumn{2}{|l|}{ Sulfasalazine } \\
\hline Patient 3 & 30 & M & 6 & \multicolumn{2}{|l|}{ MTX + sulfasalzine } \\
\hline Patient 4 & 28 & M & 8 & & \\
\hline Patient 6 & 57 & M & 15 & & \\
\hline Patient 7 & 31 & M & 3 & & \\
\hline Patient 8 & 30 & M & 3 & \multicolumn{2}{|l|}{ MTX + sulfasalzine } \\
\hline Patient 9 & 30 & M & 5 & \multicolumn{2}{|l|}{ MTX } \\
\hline Patient 10 & 27 & M & 10 & \multicolumn{2}{|l|}{ MTX } \\
\hline Patient 11 & 34 & $\mathrm{~F}$ & 1.1 & & \\
\hline Patient 12 & 35 & $\mathrm{~F}$ & 8 & \multicolumn{2}{|l|}{ Sulfasalazine } \\
\hline Patient 13 & 59 & M & 7 & \multicolumn{2}{|c|}{ Sulfasalazine + thalidomide } \\
\hline Mean & 36.2 & & 6.8 & & \\
\hline SD & 10.9 & & 4.3 & & \\
\hline Median & 31.0 & & 6.5 & & \\
\hline Designation & BASDAI & BASFI & BASG & Physician assessment & Spinal pain \\
\hline Patient 1 & 49.5 & 43 & 50 & 54 & 62 \\
\hline Patient 2 & 45.4 & 30.8 & 45 & 50 & 48 \\
\hline Patient 3 & 49.07 & 30.4 & 46 & 50 & 72 \\
\hline Patient 4 & 44.75 & 42.5 & 57 & 60 & 45 \\
\hline Patient 6 & 44.65 & 42.7 & 45 & 50 & 58 \\
\hline Patient 7 & 54.4 & 41.2 & 50 & 54 & 65 \\
\hline Patient 8 & 70 & 45.1 & 45 & 50 & 65 \\
\hline Patient 9 & 45.8 & 53.9 & 72 & 60 & 64 \\
\hline Patient 10 & 80.4 & 59.3 & 85 & 82 & 82 \\
\hline Patient 11 & 46.25 & 34.5 & 35 & 35 & 0 \\
\hline Patient 12 & 48.5 & 31.9 & 31 & 30 & 75 \\
\hline Patient 13 & 44.9 & 59.5 & 72 & 70 & 70 \\
\hline Mean & 52.0 & 42.9 & 52.8 & 53.8 & 58.83 \\
\hline SD & 11.4 & 10.3 & 16.0 & 13.9 & 21.28 \\
\hline Median & 47.4 & 42.6 & 48.0 & 52.0 & 64.5 \\
\hline Designation & ESR & CRP & Swollen joints & Tragus to wall distance & Schober test \\
\hline Patient 1 & 15 & 4.2 & 0 & 20 & 3 \\
\hline Patient 2 & 46 & 13.09 & 0 & 17 & 2 \\
\hline Patient 3 & 16 & 6.02 & 0 & 17 & 2.5 \\
\hline Patient 4 & 58 & 26.6 & 0 & 15 & 2.5 \\
\hline Patient 6 & 10 & 1 & 0 & 14 & 2.5 \\
\hline Patient 7 & 8 & 6.33 & 1 & 10 & 4 \\
\hline Patient 8 & 43 & 10 & 0 & 10 & 2.5 \\
\hline Patient 9 & 10 & 8.7 & 2 & 18 & 1 \\
\hline Patient 10 & 14 & 11.2 & 1 & 15 & 1 \\
\hline Patient 11 & 33 & 5.1 & 6 & 10 & 4.5 \\
\hline Patient 12 & 34 & 6.07 & 0 & 12 & 3.5 \\
\hline Patient 13 & 34 & 6.07 & 0 & 10 & 2 \\
\hline Mean & 26.8 & 8.7 & 0.83 & 14.00 & 2.58 \\
\hline SD & 16.7 & 6.5 & 1.75 & 3.57 & 1.06 \\
\hline Median & 24.5 & 6.2 & 0 & 14.5 & 2.5 \\
\hline
\end{tabular}




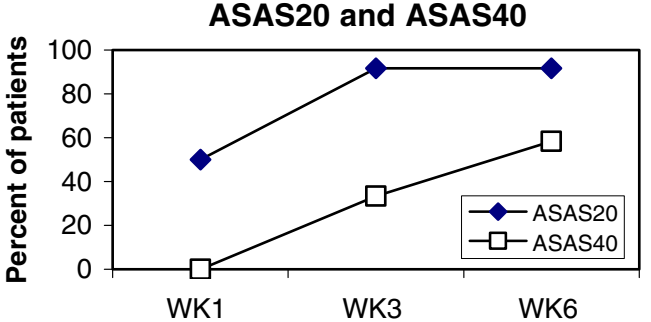

Fig. 1 Percent of patients achieving ASAS20 or ASAS40 in response to treatment by Tripterygium

[19-21]. Hence, the observation that about $90 \%$ of patients on Tripterygium achieved ASAS20 at week 6 is very encouraging. Because such comparisons across studies provide very little degree of confidence, we also attempted to document the response to Tripterygium more objectively using the acute phase reactants. There appears to be statistically significant improvement in the serum CRP, with some suppression also in ESR, although not to a statistically less significant degree.
Table 2 Changes in disease status in patients on placebo

\begin{tabular}{lll}
\hline & Week 0 & Week 6 \\
\hline BASDAI & $57.3 \pm 11.2$ & $44.7 \pm 17.4$ \\
BASFI & $50.4 \pm 21.1$ & $45.1 \pm 24.8$ \\
BAS-G & $72.8 \pm 21.1$ & $60.3 \pm 19.4$ \\
\hline
\end{tabular}

Scores are in scale $0-100$

Changes in BASDAI and BAS-G were statistically significant

Each of our patients was also questioned for possible drug-related side effects. Surprisingly, none of the patients complained of side effect, perhaps because this drug is widely accepted by patients as a standard therapy in a traditional medicine hospital. Also, perhaps because of the short duration of the study, none of the three female patients reported the expected amenorrhea. From numerous reports in Chinese journals, the most serious side effect of Tripterygium is that on fertility. In one study of 97 women treated for dermatological diseases, hypomenorrhea was observed in 39 patients, and amenorrhea in 9. There are less
Fig. 2 Changes in parameters in response to Tripterygium. Error bars represent standard deviations. The $p$ values shown are in comparison to corresponding values at week 0 . Where $p$ values are not shown, they are more than 0.05 . All $p$ values have been corrected for multiple testing. Numbers inside squares represent median values of the corresponding points
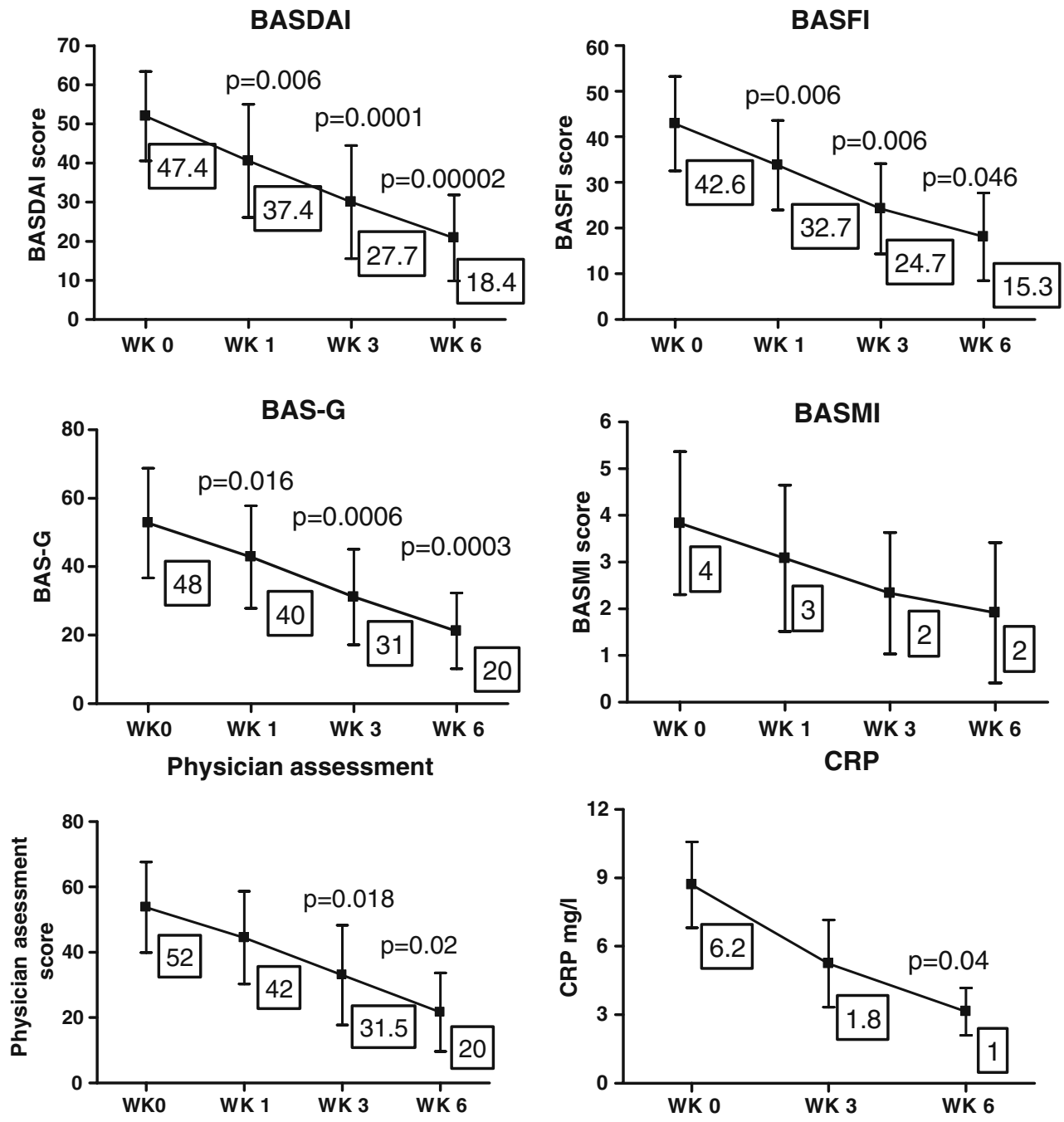
documentations of the effect of Tripterygium on male fertility. In the same study on dermatological diseases, abnormalities of sperm were detected in 24 of 66 male patients. All these occur within 3-5 weeks after starting Tripterygium [22]. There is no assurance that this adverse effect on fertility is not permanent. In addition to adverse effect for fertility, the frequency of liver toxicity has been reported to be as high as $30 \%$ and marrow suppression to be $15 \%$ [18]. We did not observe these side effects in any of our 12 patients perhaps because of the brevity of the study.

Our results carry a high risk of observer and patient bias. The high degree of response might reflect such errors in a culture where Tripterygium has been accepted as standard therapy. Based on the reports on toxicity, Tripterygium should not be initiated for any diseases by physicians with no experience with this medication. AS patients should regard the usefulness of Tripterygium with skepticism. For it to be continued to be used in AS in any country, a placebo-controlled study should be carried out to evaluate the risk/benefit ratio. At the minimum, our results can provide some basis for power analysis for such future studies.

Acknowledgment We gratefully acknowledge the assistance of Dr. Wenfeng Tan in co-coordinating this project. The project is supported by The Leading Talent Project of Traditional Chinese Medicine of Jiangsu Province.

\section{Disclosures None}

Open Access This article is distributed under the terms of the Creative Commons Attribution Noncommercial License which permits any noncommercial use, distribution, and reproduction in any medium, provided the original author(s) and source are credited.

\section{References}

1. Brinier AM, Ma J, Lipsky PE, Raskin I (2007) Medicinal chemistry and pharmacology of genus Tripterygium (Celastraceae). Phytochemistry 68(6):732-766

2. Lipsky PE, Tao XL (1997) A potential new treatment for rheumatoid arthritis: thunder god vine. Semin Arthritis Rheum 26(5):713-723

3. Zhang X, Chi Z (2004) Lei gong teng zai feng shi xing ji bing zhong de ying yong gai shu. Henan Tad Chin Med 24(3):80-82

4. Canter PH, Lee HS, Ernst E (2006) A systematic review of randomised clinical trials of Tripterygium wilfordii for rheumatoid arthritis. Phytomedicine 13(5):371-377

5. Goldbach-Mansky R, Wilson M, Fleischmann R, Olsen N, Silverfield J, Kempf P et al (2009) Comparison of Tripterygium wilfordii Hook $\mathrm{F}$ versus sulfasalazine in the treatment of rheumatoid arthritis: a randomized trial. Ann Intern Med 151 (4):229-240
6. Tao X, Younger J, Fan FZ, Wang B, Lipsky PE (2002) Benefit of an extract of Tripterygium wilfordii Hook $\mathrm{F}$ in patients with rheumatoid arthritis: a double-blind, placebo-controlled study. Arthritis Rheum 46(7):1735-1743

7. Tao XL, Dong Y, Zhang NZ (1987) A double-blind study of T2 (tablets of polyglycosides of Tripterygium wilfordii Hook) in the treatment of rheumatoid arthritis. Zhonghua Nei Ke Za Zhi 26:399-402

8. Guo J, Yuan S, Wang X, Xu S, Li D (1981) Tripterygium wilfordii Hook $\mathrm{f}$ in rheumatoid arthritis and ankylosing spondylitis. Preliminary report. Chin Med J 94(7):405-412

9. Sieper J, Rudwaleit M, Khan MA, Braun J (2006) Concepts and epidemiology of spondyloarthritis. Best Pract Res Clin Rheumatol 20(3):401-417

10. Zochling J (2008) Assessment and treatment of ankylosing spondylitis: current status and future directions. Curr Opin Rheumatol 20(4):398-403

11. van der Linden S, Valkenburg HA, Cats A (1984) Evaluation of diagnostic criteria for ankylosing spondylitis. A proposal for modification of the New York criteria. Arthritis Rheum 27 (4):361-368

12. Pang L, Wang L, Suo T, Hao H, Fang X, Jia J et al (2008) Tumor necrosis factor-alpha blockade leads to decreased peripheral $\mathrm{T}$ cell reactivity and increased dendritic cell number in peripheral blood of patients with ankylosing spondylitis. J Rheumatol 35 (11):2220-2228

13. Calin A, Garrett S, Whitelock H, Kennedy LG, O'Hea J, Mallorie P et al (1994) A new approach to defining functional ability in ankylosing spondylitis: the development of the bath ankylosing spondylitis functional index. J Rheumatol 21 (12):2281-2285

14. Garrett S, Jenkinson T, Kennedy LG, Whitelock H, Gaisford P, Calin A (1994) A new approach to defining disease status in ankylosing spondylitis: the bath ankylosing spondylitis disease activity index. J Rheumatol 21(12):2286-2291

15. Jenkinson TR, Mallorie PA, Whitelock HC, Kennedy LG, Garrett SL, Calin A (1994) Defining spinal mobility in ankylosing spondylitis (AS). The bath as metrology index. J Rheumatol 21 (9):1694-1698

16. Jones SD, Steiner A, Garrett SL, Calin A (1996) The bath ankylosing spondylitis patient global score (BAS-G). Br J Rheumatol 35(1):66-71

17. Anderson JJ, Baron G, van der Heijde D, Felson DT, Dougados M (2001) Ankylosing spondylitis assessment group preliminary definition of short-term improvement in ankylosing spondylitis. Arthritis Rheum 44(8):1876-1886

18. Wan $\mathrm{T}$ (2005) The adverse reaction of lei gong teng. Pract Clin Med 6:149-150

19. Inman RD, Davis JC Jr, Heijde DV, Diekman L, Sieper J, Kim SI et al (2008) Efficacy and safety of golimumab in patients with ankylosing spondylitis: results of a randomized, double-blind, placebocontrolled, phase III trial. Arthritis Rheum 58(11):3402-3412

20. van der Heijde D, Dijkmans B, Geusens P, Sieper J, DeWoody K, Williamson P et al (2005) Efficacy and safety of infliximab in patients with ankylosing spondylitis: results of a randomized, placebo-controlled trial (ASSERT). Arthritis Rheum 52(2):582-591

21. van der Heijde D, Schiff MH, Sieper J, Kivitz A, Wong RL, Kupper H, et al (2008) Adalimumab effectiveness for the treatment of ankylosing spondylitis is maintained for up to 2 years: long-term results from the ATLAS trial. Ann Rheum Dis

22. Tang Y, Zhang X, Su B (1998) The adverse reaction of lei gong teng application in dermatology. J Clin Dermatol 27:377 